\title{
Sciendo
}

Int. J. of Applied Mechanics and Engineering, 2019, vol.24, No.2, pp.259-268

DOI: 10.2478-ijame-2019-0017

\section{STUDY OF AXI-SYMMETRIC VIBRATIONS IN A MICROPOLAR TRANSVERSELY ISOTROPIC LAYER}

\author{
R.R. GUPTA* and R.R. GUPTA \\ Department of Mathematics and Applied Sciences, MEC \\ Muscat, OMAN \\ E-mail: *dr.rajanigupta@gmail.com; raji.mmec@gmail.com
}

\begin{abstract}
The present investigation deals with the propagation of circular crested Lamb waves in a homogeneous micropolar transversely isotropic medium. Secular equations for symmetric and skew-symmetric modes of wave propagation in completely separate terms are derived. The amplitudes of displacements and microrotation are computed numerically for magnesium as a material and the dispersion curves, amplitudes of displacements and microrotation for symmetric and skew-symmetric wave modes are presented graphically to evince the effect of anisotropy. Some special cases of interest are also deduced.
\end{abstract}

Key words: micropolar, transversely isotropic, amplitude ratios.

\section{Introduction}

Classical mechanics deals with the basic assumption that the effect of the microstructure of a material is not essential for describing mechanical behavior. Such an approximation has been shown in many well-known cases. Often, however, discrepancies between the classical theory and experiments are observed, indicating that the microstructure might be important. For example, discrepancies have been found in the stress concentrations in the areas of holes, notches and cracks; elastic vibrations characterized by a high frequency and small wavelengths, particularly in granular composites consisting of stiff inclusions embedded in a weaker matrix, fibers or grains; and the mechanical behavior of complex fluids such as liquid crystals, polymeric suspensions, and animal blood. In general, granular composites, for example porous materials, are widely used in the area of passive noise control as sound absorbers. The effect of acoustical waves characterized by high frequencies and small wavelengths become significant.

To explain the fundamental departure of microcontinuum theories from the classical continuum theories, a continuum model embedded with microstructures to describe the microscopic motion or a non local model to describe the long range material interaction are developed. This theory extends the application of the continuum model to microscopic space and short-time scales. The micromorphic theory $[1,2]$ treats a material body as a continuous collection of a large number of deformable particles, with each particle possessing finite size and inner structure. Using assumptions such as infinitesimal deformation and slow motion, the micromorphic theory can be reduced to Mindlin's [3] microstructure theory. When the microstructure of the material is considered rigid, it leads the micropolar theory [4].

Eringen's micropolar theory is more appropriate for geological materials such as rocks, soils since, this theory takes into account the intrinsic rotation and predicts the behavior of a material with an inner structure.

There has been considerable interest generated in recent years in wave propagation through naturally occurring media and man-made materials in view of widespread applications in acoustic signal transmissions, seismically induced motions, non-destructive evaluation, noise control, subsurface exploration, etc. Cylindrical plates are frequently used as structural components and their vibration

\footnotetext{
* To whom correspondence should be addressed
} 
characteristics are important for practical design. The waves which propagate in a freely vibrating plate are called Lamb waves. The unique properties of Lamb waves have made them increasingly attractive for nondestructive testing of bonded structures. The sensitivity and effciency of adhesive bond inspection using Lamb waves have been the subject of study in recent years in many laboratories concerned with bond quality inspection. Different researchers discussed different types of problems in transversely isotropic elastic materials. Abubakar [5] discussed free vibrations of a transversely isotropic plate. Keck et al. [6] derived the frequency equation for the propagation of train of nontorsional axisymmetric harmonic wave in infinitely long shells, made of three concentric cylinders of different transversely isotropic materials. Shuvalov et al. [7] described long wavelength onset of the fundamental branches for a free anisotropic plate. Payton [8] in 1991 studied wave propagation in a restricted transversely isotropic elastic solid whose slowness surface contains conical points. However, no attempt has been made to study wave propagation in a micropolar transversely isotropic medium.

The aim of the present study is to enhance our knowledge about the propagation of axisymmetric waves in the layer of a micropolar transversely isotopic material. This study has many applications in various fields of science and technology, namely, atomic physics, industrial engineering, thermal power plants, submarine structures, pressure vessel, aerospace, chemical pipes and metallurgy. After developing the solution, frequency equations connecting the phase velocity with wave number, for symmetric and skewsymmetric wave modes are derived. The amplitude ratios of displacements and microrotation are also obtained. To indicate the effect of anisotropy, the dispersion curves, attenuation coeffcients, amplitude ratio of displacements and microrotation for symmetric and skew-symmetric waves are presented and illustrated graphically.

\section{Basic equations}

Following Eringen [9], the constitutive relations and balance laws in a general micropolar anisotropic medium possessing center of symmetry, in the absence of body forces, body couples, are given by

\section{constitutive relations:}

$$
\begin{aligned}
& t_{i j}=A_{i j k l} e_{k l}+G_{i j k l} \chi_{k l}, \\
& m_{i j}=G_{i j k l} E_{k l}+B_{i j k l} \chi_{k l} .
\end{aligned}
$$

The deformation and wryness tensor are defined by

$$
E_{j i}=u_{i, j}+\varepsilon_{i j k l} \varphi_{k}, \quad \chi_{i j}=\varphi_{i, j}
$$

balance laws

$$
\begin{aligned}
& t_{i j, j}=\rho \ddot{u}_{i}, \\
& m_{i k, i}+\varepsilon_{i j k} t_{i j}=\rho j \ddot{\varphi}_{k}
\end{aligned}
$$

where the list of symbols is given in the nomenclature.

\section{Problem formulation}

We have used appropriate transformations following Slaughter [10], on the set of Eqs (2.1) to derive equations for a micropolar transversely isotopic medium and restricted our analysis to the two dimensional problem. 
In the present paper, we consider an infinite layer with traction free surfaces at $z= \pm H$ (layer of thickness $2 H$ ), which consists of a homogeneous, micropolar transversely isotropic material. The plate is axisymmetric with respect to the $z$-axis as the axis of symmetry. We take the origin of the co-ordinate system $(r, \theta, z)$ on the middle surface of the plate and the $z$-axis is taken normal to the solid plate along the thickness. We take the $r-z$ plane as the plane of incidence. If we restrict our analysis to the plane strain problem parallel to the $r-z$ plane with the displacement vector $\boldsymbol{u}=\left(u_{r}, 0, u_{z}\right)$, microrotation vector $\varphi=\left(0, \varphi_{\theta}, 0\right)$ and $\partial / \partial \theta=0$, so that the field equations and constitutive relations in cylindrical polar coordinates reduce to

$$
\begin{aligned}
& A_{11}\left(\frac{\partial^{2} u_{r}}{\partial r^{2}}+\frac{1}{r} \frac{\partial u_{r}}{\partial r}-\frac{u_{r}}{r^{2}}\right)+A_{55} \frac{\partial^{2} u_{r}}{\partial z^{2}}+\left(A_{13}+A_{56}\right) \frac{\partial^{2} u_{z}}{\partial r \partial z}+K_{1} \frac{\partial \varphi_{\theta}}{\partial z}=\rho \frac{\partial^{2} u_{r}}{\partial t^{2}} \\
& A_{66}\left(\frac{\partial^{2} u_{z}}{\partial r^{2}}+\frac{1}{r} \frac{\partial u_{z}}{\partial z}\right)+A_{33} \frac{\partial^{2} u_{z}}{\partial z^{2}}+\left(A_{13}+A_{56}\right)\left(\frac{\partial^{2} u_{r}}{\partial r \partial z}+\frac{1}{r} \frac{\partial u_{r}}{\partial z}\right)+K_{2}\left(\frac{\partial \phi_{\theta}}{\partial r}+\frac{\phi_{\theta}}{r}\right)=\rho \frac{\partial^{2} u_{z}}{\partial t^{2}} \\
& B_{77}\left(\frac{\partial^{2} \varphi_{\theta}}{\partial r^{2}}+\frac{1}{r} \frac{\partial \varphi_{\theta}}{\partial r}-\frac{\varphi_{\theta}}{r^{2}}\right)+B_{66} \frac{\partial^{2} \varphi_{\theta}}{\partial z^{2}}-X \varphi_{\theta}-K_{1} \frac{\partial u_{r}}{\partial z}-K_{2} \frac{\partial u_{z}}{\partial r}=\rho j \frac{\partial^{2} \varphi_{\theta}}{\partial t^{2}}
\end{aligned}
$$

where

$$
K_{1}=A_{56}-A_{55}, \quad K_{2}=A_{66}-A_{56}, \quad X=K_{2}-K_{1},
$$

and we have used the notations $11 \rightarrow 1,33 \rightarrow 3,12 \rightarrow 7,13 \rightarrow 6,23 \rightarrow 5$ for the material constants.

For further considerations, it is convenient to introduce the dimensionless variables defined by

$$
\begin{aligned}
& \left(r^{\prime}, z^{\prime}\right)=\frac{\omega^{*}}{c_{1}}(r, z), \quad\left(u_{r}^{\prime}, u_{z}^{\prime}\right)=\frac{\omega^{*}}{c_{1}}\left(u_{r}, u_{z}\right), \quad t_{i j}^{\prime}=\frac{t_{i j}}{A_{55}}, \quad m_{i j}^{\prime}=\frac{m_{i j} c_{1}}{B_{77} \omega^{*}}, \\
& \varphi_{\theta}^{\prime}=\frac{\varphi_{\theta} A_{55}}{K_{1}}, \quad t^{\prime}=\omega^{*} t, \quad \omega^{* 2}=\frac{X}{\rho j}, \quad c_{1}^{2}=\frac{A_{55}}{\rho} .
\end{aligned}
$$

\section{Boundary condition}

The boundaries of the layer are assumed to be stress free. Therefore, at the surfaces $z= \pm H$, the appropriate boundary conditions are

$$
t_{z z}=0, \quad t_{z r}=0, \quad m_{z \theta}=0
$$

where $t_{z z}, t_{z r}$ are the normal and tangential stress components, $m_{z \theta}$ is the tangential couple stress, whose values in the present case are defined as

$$
\begin{aligned}
& t_{z z}=A_{13}\left(\frac{\partial u_{r}}{\partial r}+\frac{u_{r}}{r}\right)+A_{33} \frac{\partial u_{z}}{\partial z} \\
& t_{z r}=A_{65}\left(\frac{\partial u_{z}}{\partial r}+\phi_{\theta}\right)+A_{55}\left(\frac{\partial u_{r}}{\partial r}-\phi_{\theta}\right), \\
& m_{z \theta}=B_{66} \frac{\partial \phi_{\theta}}{\partial z}
\end{aligned}
$$




\section{Normal mode analysis and solution of the problem}

We assume the solution for $u_{r}, u_{z}, \varphi_{\theta}$ representing propagating waves in the $r-z$ plane of the form

$$
\left(u_{r}, u_{z}, \varphi_{\theta}\right)=\left(J_{l}(\xi r), \bar{u}_{z} J_{o}(\xi r), \bar{\varphi}_{\theta}, J_{l}(\xi r)\right) u_{r} e^{i \xi(m z-c t)}
$$

where $\xi$ is the wave number, $\omega=\xi c$ is the angular frequency and $c$ is the phase velocity of the wave, $m$ is the unknown parameter which signifies the penetration depth of the wave, $\bar{u}_{z}, \bar{\varphi}_{\theta}$ are respectively, the amplitude ratios of the displacement $u_{z}$, microrotation $\phi_{\theta}$ to that of the displacement $u_{r}$.

With the help of Eqs (3.4) and (5.1), Eqs (3.1)-(3.3) reduce to (after suppressing primes)

$$
\begin{aligned}
& -m^{2} \xi^{2}+\left(c_{1}-d_{1}\right) \xi^{2}-i \xi^{2} m d_{2} \bar{u}_{z}+i \xi m d_{3} \bar{\varphi}_{\theta}=0, \\
& m i \xi^{2} d_{5}+\xi^{2}\left(-m^{2} d_{7}-d_{4}+c^{2}\right) \bar{u}_{z}+\xi d_{6} \bar{\varphi}_{\theta}=0, \\
& -i \xi m d_{9}-\xi d_{10} \bar{u}_{z}+\left[d_{11}-\left(m^{2}+d_{8}-c^{2} d_{12}\right) \xi^{2}\right] \bar{\varphi}_{\theta}=0
\end{aligned}
$$

where

$$
\begin{aligned}
& d_{1}=A_{11} / A_{55}, \quad d_{2}=d_{5}=\left(A_{13} /+A_{56}\right) / A_{55}, \quad d_{3}=K_{1}^{2} / A_{55}^{2}, \quad d_{4}=A_{66} / A_{33}, \\
& d_{6}=K_{1} K_{2} / A_{33} A_{55}, \quad d_{7}=A_{55} / A_{33}, \quad d_{8}=B_{77} / B_{66}, \quad d_{9}=-A_{55} c_{1}^{2} / B_{66} \omega^{* 2}, \\
& d_{10}=-K_{2} A_{55} c_{1}^{2} / K_{1} B_{66} \omega^{* 2}, \quad d_{11}=-X c_{1}^{2} / B_{66} \omega^{* 2}, \quad d_{12}=A_{55} j / B_{66}, \quad d_{13}=A_{13} / A_{55} .
\end{aligned}
$$

The condition for the non trivial solution of the system of Eqs (5.2), yields a cubic equation in $q\left(=m^{2}\right)$ as

where

$$
A m^{6}+B m^{4}+C m^{2}+D=0
$$

$$
\begin{aligned}
& A=-\xi^{6} d_{7}, \quad B=-\xi^{4}\left(-d_{7} d_{8}+d_{4}-d_{1} d_{7}\right)-\xi^{3} d_{5}^{2} d_{12}\left(\omega^{2}+1\right)+ \\
& +\xi^{5} d_{8} d_{5}^{2}-\xi^{2} \omega^{2}+\xi^{2} d_{7}\left(\omega^{2} d_{12}+d_{11}-d_{3} d_{9}+\omega^{2}\right), \\
& C=\xi^{2}\left(d_{6} d_{10}-\omega^{2} d_{8}-d_{4} \omega^{2} d_{12}+d_{4} d_{11}-\omega^{2} c^{2} d_{12}-c^{2} d_{11}-d_{3} d_{5} d_{10}+d_{3} d_{9}\left(d_{4}-c^{2}\right)+\right. \\
& \left.+d_{2} d_{6} d_{9}\right)+d_{8} d_{4} \xi^{2}-\xi^{2}\left(\xi^{2} d_{1}-\omega^{2}\right)\left(\xi^{2} d_{7} d_{8}-d_{7}\left(\omega^{2} d_{12}+d_{11}\right)-\xi^{2}\left(d_{4}-c^{2}\right)\right), \\
& D=\left(\xi^{2} d_{1}-\omega^{2}\right)\left[\xi^{2} d_{6} d_{10}+\xi^{2}\left(d_{4}-c^{2}\right)\left(d_{8} \xi^{2}-\omega^{2} d_{12}-d_{11}\right)\right] .
\end{aligned}
$$

The roots of this equation give three values of $m^{2}$, and hence of $c^{2}$. Three positive values of $c$ will be the velocities of propagation of three possible waves, viz. the quasi-longitudinal displacement (QLD) wave, transverse displacement (QCTD) wave and quasi-coupled quasi-coupled transverse microrotational 
(QCTM) wave. This fact is verified, when we solve Eq.(5.4), using Matlab programming. For a isotropic linear micropolar elastic solid, i.e., if we put

$$
\begin{aligned}
& A_{11}=A_{33}=\lambda+2 \mu+K, \quad A_{55}=A_{66}=\mu+K, \quad A_{13}=\lambda, A_{56}=\mu, \\
& K=-K_{1}=K_{2}=X / 2, \quad B_{66}=B_{77}=\gamma,
\end{aligned}
$$

in Eq.(5.4), the velocity $c_{1}$ corresponds to the longitudinal displacement wave and the velocities $c_{2}$ and $c_{3}$ correspond to two coupled waves, viz. the transverse microrotational and transverse displacement wave as obtained by Parfitt and Eringen [11].

So Eq.(5.4) leads to the following solution for displacements and microrotation as

$$
\left(\bar{u}_{r}, \bar{u}_{z}, \bar{\varphi}_{\theta}\right)=\sum_{k=1}^{3}\left[A_{k} \cos \left(m_{k} \xi z\right)+B_{k} \sin \left(m_{k} \xi\right)\right]\left(J_{l}(\xi r), r_{k} J_{o}(\xi r), t_{k} J_{l}(\xi r)\right) e^{i \omega t}
$$

where

$$
\left(r_{k}, t_{k}\right)=-m_{k} \frac{\left(\left[-m_{k}^{2} \xi^{2} d_{7}-d_{5} d_{10} \xi^{2}+b_{1}\right], \xi\left[m_{k}^{2} \xi^{2} d_{5}+b_{2} d_{5}+d_{6} d_{9}\right]\right)}{m_{k}^{4} \xi^{2} d_{7}+m_{k}^{2} \xi^{2}\left(d_{7} b_{2}-b_{1}\right)-\xi^{2} d_{6} d_{10}-b_{1} b_{2}} .
$$

\section{Derivation of secular equation}

Substituting the values of $\bar{u}_{r}, \bar{u}_{z}$ and $\bar{\varphi}_{\theta}$ in the boundary conditions (4.1) at the surfaces $\pm H$ of the layer, yields

$$
\begin{aligned}
& \sum_{k=1}^{3}\left[\left(g_{1} c_{k}-g_{2 k} s_{k}\right) A_{k}+\left(g_{1} s_{k}+g_{2 k} c_{k}\right) B_{k}\right]=0, \\
& \sum_{k=1}^{3}\left[\left(g_{1} c_{k}+g_{2 k} s_{k}\right) A_{k}+\left(-g_{1} s_{k}+g_{2 k} c_{k}\right) B_{k}\right]=0, \\
& \sum_{k=1}^{3}\left[\left(g_{3 k} c_{k}-g_{4 k} s_{k}\right) A_{k}+\left(g_{3 k} s_{k}+g_{4 k} c_{k}\right) B_{k}\right]=0, \\
& \sum_{k=1}^{3}\left[\left(g_{3 k} c_{k}+g_{4 k} s_{k}\right) A_{k}+\left(-g_{3 k} s_{k}+g_{4 k} c_{k}\right) B_{k}\right]=0, \\
& \sum_{k=1}^{3}\left[-g_{5 k} s_{k} A_{k}+g_{5 k} c_{k} B_{k}\right]=0, \quad \sum_{k=1}^{3}\left[g_{5 k} s_{k} A_{k}+g_{5 k} c_{k} B_{k}\right]=0
\end{aligned}
$$

where

$$
\begin{aligned}
& s_{k}=\sin \left(m_{k} \xi H\right), \quad c_{k}=\cos \left(m_{k} \xi H\right), \quad g_{1}=d_{13} \xi, \quad g_{2 k}=\frac{m_{k} r_{k}}{d_{7}}, \quad g_{3 k}=-m_{k} \xi, \\
& g_{4 k}=-\xi d_{4} r_{k}+d_{15} t_{k}, \quad g_{5 k}=m_{k} \xi t_{k}, \quad k=1,2,3 .
\end{aligned}
$$


In order that the six boundary conditions given by Eqs (4.1) be satisfied simultaneously, the determinant of the coefficients of $A_{k}$ and $B_{k} k=1,2,3$ in Eqs (6.1) vanishes. This gives an equation for the frequency of the layer oscillations. The frequency equation for the waves in the present case, after applying lengthy algebraic reductions and manipulations of the determinant leads to the following secular equation

$$
\left[T_{1}\right]^{ \pm} g_{1} g_{51}\left(g_{33}-g_{32}\right)+\left[T_{2}\right]^{ \pm} g_{1} g_{52}\left(g_{31}-g_{33}\right)+\left[T_{3}\right]^{ \pm} g_{1} g_{53}\left(g_{32}-g_{31}\right)=0
$$

These are the frequency equations which correspond to the symmetric and skew symmetric mode with respect to the medial plane $z=0$. Here, the superscript ' + ' corresponds to skew symmetric and '-' refers to symmetric modes and

$$
T_{k}=\tan \left(m_{k} \xi z\right), \quad k=1,2,3 .
$$

\subsection{Specific loss}

The specific loss is the ratio of energy $(\Delta W)$ dissipated in taking a specimen through a stress cycle to the elastic energy $(W)$ stored in the specimen when the strain is maximum. Kolsky [12] shows that the specific loss $(\Delta W / W)$ is, $c$ times the absolute value of the ratio of the imaginary part of the wave number to the real part of the wave number i.e.

$$
\frac{\Delta W}{W}=4 \pi\left|\frac{\operatorname{Im}(k)}{\operatorname{Re}(k)}\right| .
$$

He noted that the specific loss is the most direct method of defining internal friction for a material.

\subsection{Amplitudes of displacements and microrotation}

In this section, the amplitudes of displacement components and microrotation for symmetric and skew symmetric modes of plane waves can be obtained as

$$
\begin{aligned}
& {\left[\left(\bar{u}_{1}\right)_{\text {sym }},\left(\bar{u}_{1}\right)_{\text {asym }}\right]=\sum_{k=1}^{3}\left[A_{k} \cos \left(m_{k} z \xi\right), B_{k} \sin \left(m_{k} z \xi\right)\right] e^{i \xi\left(x_{1}-c t\right)},} \\
& {\left[\left(\bar{u}_{3}\right)_{\text {sym }},\left(\bar{u}_{3}\right)_{\text {asym }}\right]=\sum_{k=1}^{3} r_{k}\left[A_{k} \cos \left(m_{k} z \xi\right), B_{k} \sin \left(m_{k} z \xi\right)\right] e^{i \xi\left(x_{1}-c t\right)},} \\
& {\left[\left(\bar{\varphi}_{2}\right)_{\text {sym }},\left(\bar{\varphi}_{2}\right)_{\text {asym }}\right]=\sum_{k=1}^{3} t_{k}\left[A_{k} \cos \left(m_{k} z \xi\right), B_{k} \sin \left(m_{k} z \xi\right)\right] e^{i \xi\left(x_{1}-c t\right)} .}
\end{aligned}
$$

\section{Numerical results and discussion}

In order to illustrate theoretical results obtained in the preceding sections, we now present some numerical results. The following relevant physical constants are chosen arbitrarily for a micropolar transversely isotopic material due to unavailability of relevant experimental data

$$
\begin{aligned}
& A_{11}=16.8 \times 10^{10} \mathrm{Nm}^{-2}, \quad A_{33}=18.43 \times 10^{10} \mathrm{Nm}^{-2}, \quad A_{55}=3.7 \times 10^{10} \mathrm{Nm}^{-2}, \quad A_{66}=4.2 \times 10^{10} \mathrm{Nm}^{-2}, \\
& A_{13}=7.85 \times 10^{10} \mathrm{Nm}^{-2}, \quad A_{56}=3.77 \times 10^{10} \mathrm{Nm}^{-2}, \quad B_{77}=5.71 \times 10^{9} \mathrm{~N}, \quad B_{66}=5.648 \times 10^{9} \mathrm{~N} .
\end{aligned}
$$


For comparison with a micropolar isotropic solid, following Eringen [13], we take the following values of relevant parameters of a micropolar isotropic solid for the case of magnesium crystal like material as

$$
\begin{array}{lll}
\rho=1.74 \times 10^{3} \mathrm{Kg} / \mathrm{m}^{3}, & \lambda=9.4 \times 10^{10} \mathrm{~N} / \mathrm{m}^{2}, & \mu=4.0 \times 10^{10} \mathrm{~N} / \mathrm{m}^{2}, \\
K=1.0 \times 10^{10} \mathrm{~N} / \mathrm{m}^{2}, & \gamma=0.779 \times 10^{-9} \mathrm{~N}, & j=0.2 \times 10^{-19} \mathrm{~m}^{2} .
\end{array}
$$

Equation (5.3) determines the phase velocity $\mathrm{c}$ of the surface waves as a function of the wave number $\xi$ and various physical parameters in complex form.

Graphical representation is given for the non-dimensional phase velocity, attenuation coeffcient and specific loss with respect to $(R)$, i.e. the real part of the wave number (Figs 1-6) restricted to thickness $H=1$ for symmetric and skew symmetric modes, to compare the results for a micropolar transversely isotropic solid (MTIS) and a micropolar isotropic solid (MIS). Here, curves represented by solid lines with and without center symbol represent the variation corresponding to MTIS and curves represented by broken lines with and without center symbol represent the variations corresponding to MIS. The curves shown in the figures without center symbol represent the variations corresponding to initial mode $(n=1)$ of wave propagation, curves with center symbol $(-O-)$ represent the variations corresponding to second mode $(n=2)$ and curves with center symbol $(-\times-)$ represent the variations corresponding to final mode $(n=3)$ of wave propagation. Figures 7-9 show the variations of amplitude of radial displacement, tangential displacement and microrotation with thickness of the layer. Figures 1 and 4 depict the variations of phase velocity with respect to $(R)$, for symmetric and skew symmetric modes, respectively. It is evident from Fig. 1 that for the first and second mode, the values of phase velocity start with a slight initial decrease and then oscillate to attain a constant value afterwards, in both the cases of MTIS and MIS. However, for the highest mode $(n=3)$ its value sharply decreases over the interval $(0,1)$, and then oscillates to attain a constant value about the origin for both the cases of MTIS and MIS. However, for the skew symmetric case as follows form Fig.4, for all the modes of wave propagation $(n=1,2,3)$ the values of phase velocity show a large hump over the interval $(0.5,4)$ and then decrease to become constant as far as the case of MTIS is considered, but in the case of MIS, the values oscillate within the range $(0, \sigma)$ and then decrease to attain a constant value in the end. The peak value of phase velocity for the skew symmetric mode is higher for the highest mode of wave propagation.

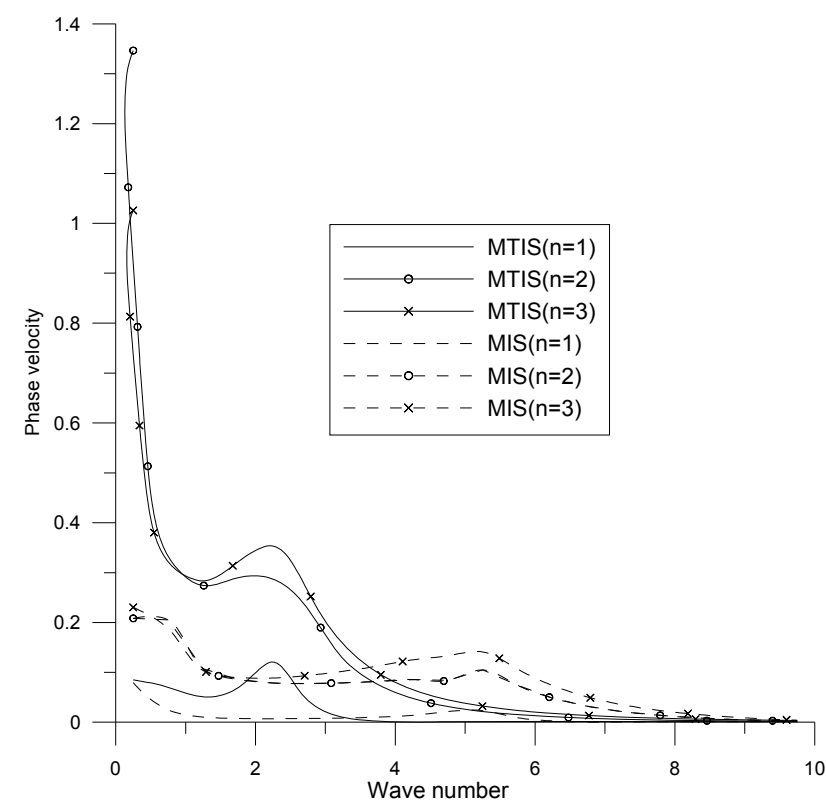

Fig.1. Variation of phase velocity with wave number for symmetric mode.

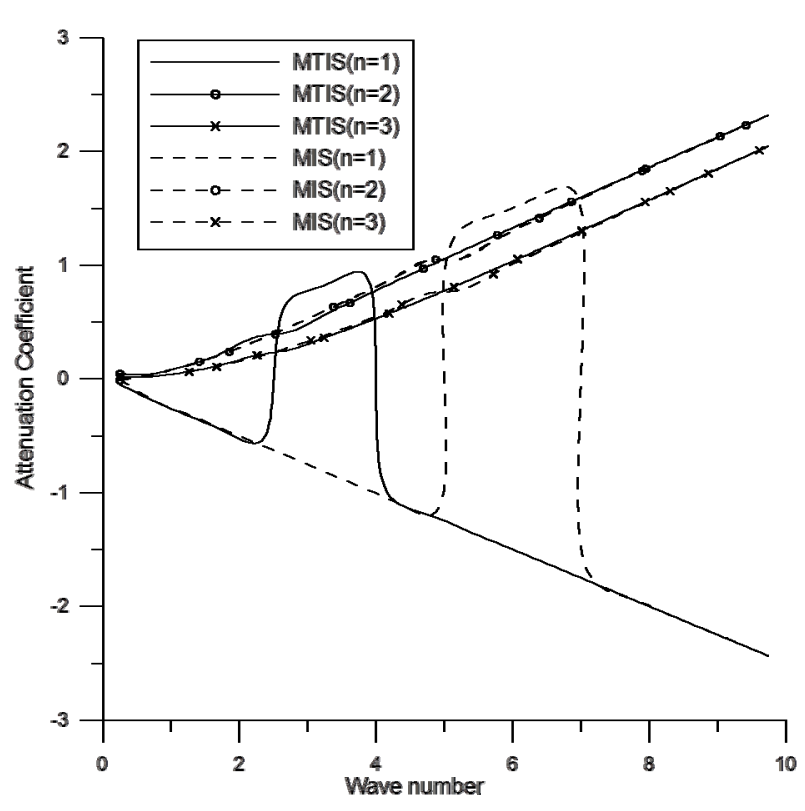

Fig.2. Variation of attenuation coefficient with wave number for symmetric mode. 


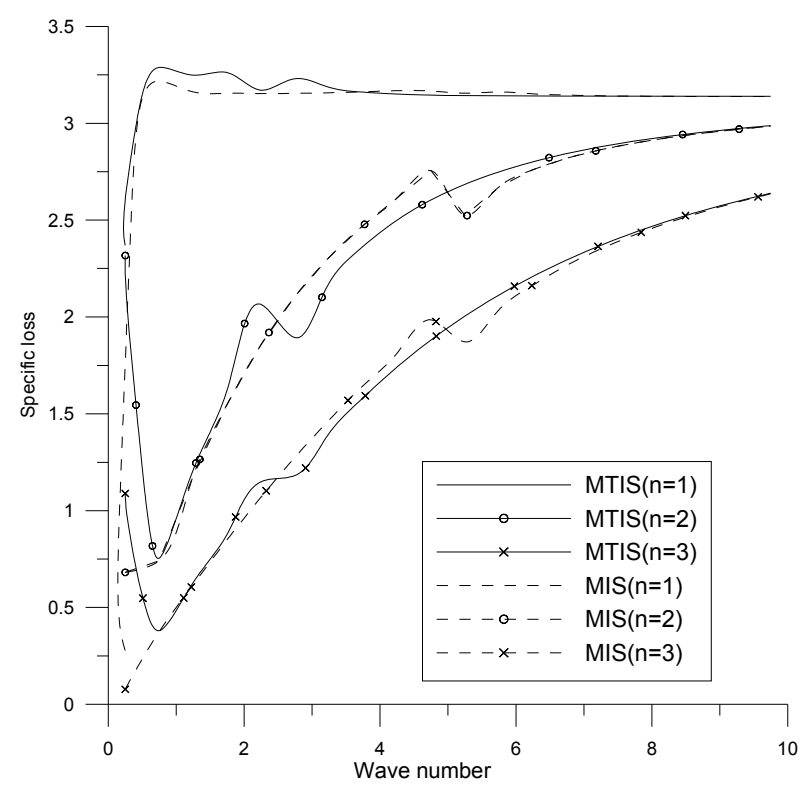

Fig.3. Variation of specific loss with wave number for symmetric mode.

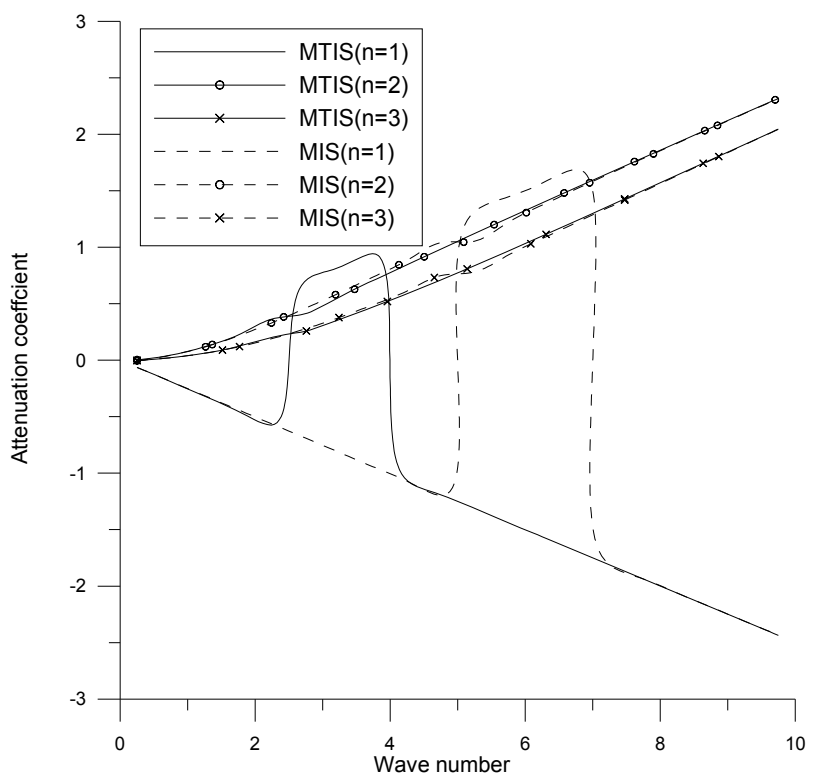

Fig.5. Variation of attenuation coefficient with wave number for skew symmetric mode.

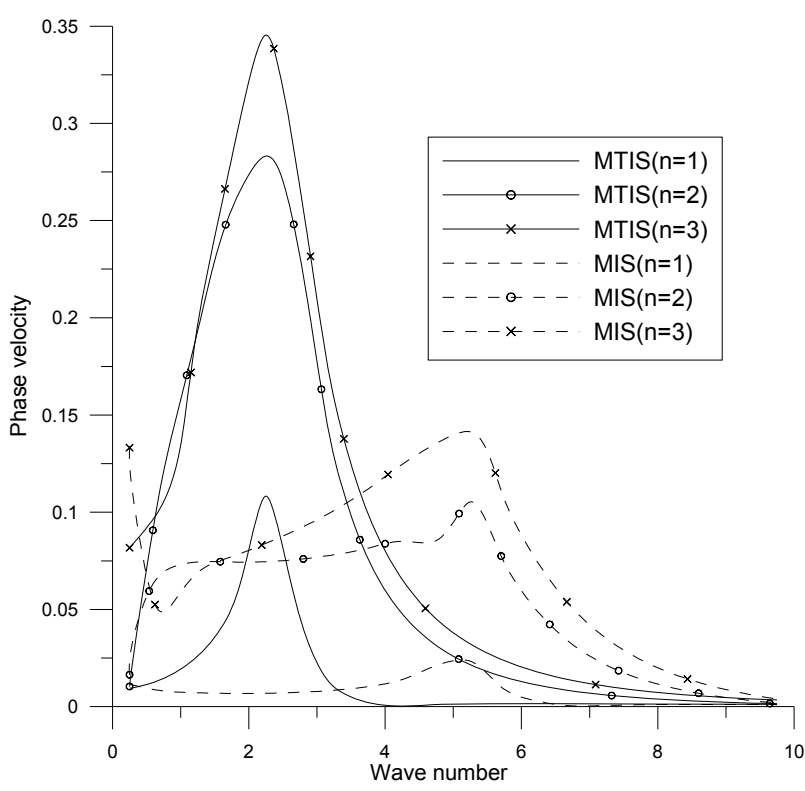

Fig.4. Variation of phase velocity with wave number for skew symmetric mode.

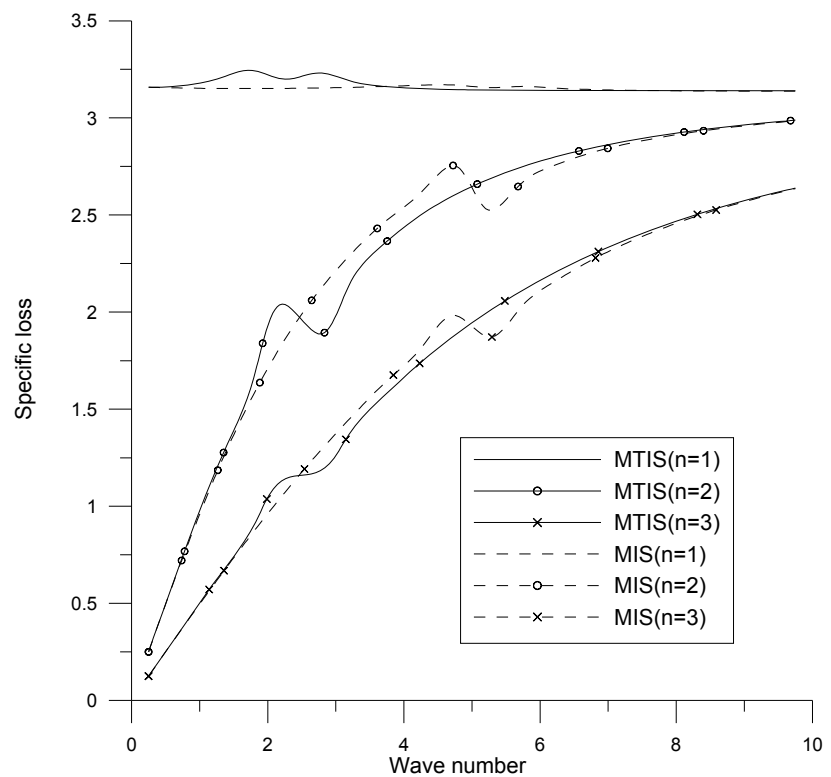

Fig.6. Variation of specific loss with wave number for skew symmetric mode.

Figures 2 and 5 indicate the variations of attenuation coeffcient for both symmetric and skew symmetric cases. It is evident from these figures that the variation pattern for both MTIS and MIS in both the figures is same. It is also observed that the value of attenuation coeffcient for the initial mode of wave propagation decreases with arbitrary oscillation, while for a higher mode, its value constantly increases. The variations of the specific loss with respect to $R$ for symmetric and skew symmetric modes can be in Figs 3 and 6, respectively. It is seen in Fig. 3 that for the initial mode the value of the specific loss initially increases and then oscillates to attain a constant value for both MTIS and MIS. As we move to a higher mode of wave propagation, its value starts with a sharp initial decrease over the interval $(0.25,0.75)$ and then oscillates to get a constant value at the end. However, in the skew symmetric case, for the initial mode of wave propagation its value oscillates with a very small amplitude and becomes constant. While for higher modes of wave 
propagation $(n=2,3)$, its value increase with oscillating amplitude to attain a constant value at the end, in both the cases of MTIS and MIS. It is observed that due to anisotropy the value of the specific loss gets increased.

Figures 7-9 indicate the trend of variations of the amplitude of radial displacement, tangential displacement and microrotation with respect to the thickness $H$ of the layer. Figures 7 and 9 show that the values of the amplitude of radial displacement and microrotation for MTIS and for symmetric mode, oscillate constantly with a small amplitude about the origin. While for the skew symmetric mode its value oscillates increasingly with small amplitudes. The value for the skew symmetric mode is higher as compared to that of the symmetric mode. It is illustrated in Fig. 8 that the value of amplitude of tangential displacement for both MTIS and MIS and for both symmetric and skew symmetric modes appears to be constant over the interval $(0,3)$ and then increases constantly. It is also observed from the figure that the value of amplitude of tangential displacement is higher for the skew symmetric mode.
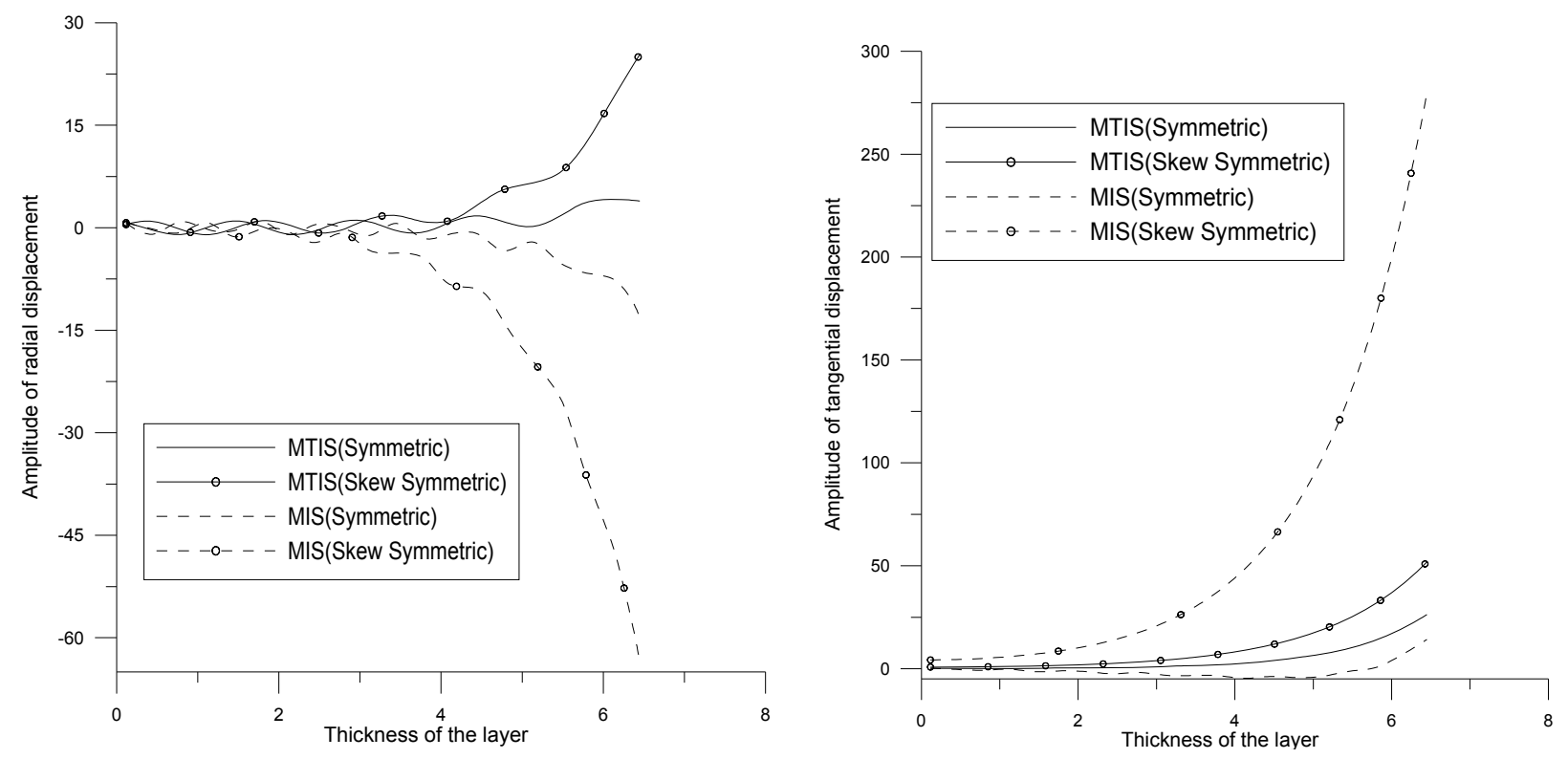

Fig.7. Variation of amplitude ratio of radial Fig.8. Variations of amplitude of tangential displacement with thickness $H$ of the layer. displacement with thickness $H$ of the layer.

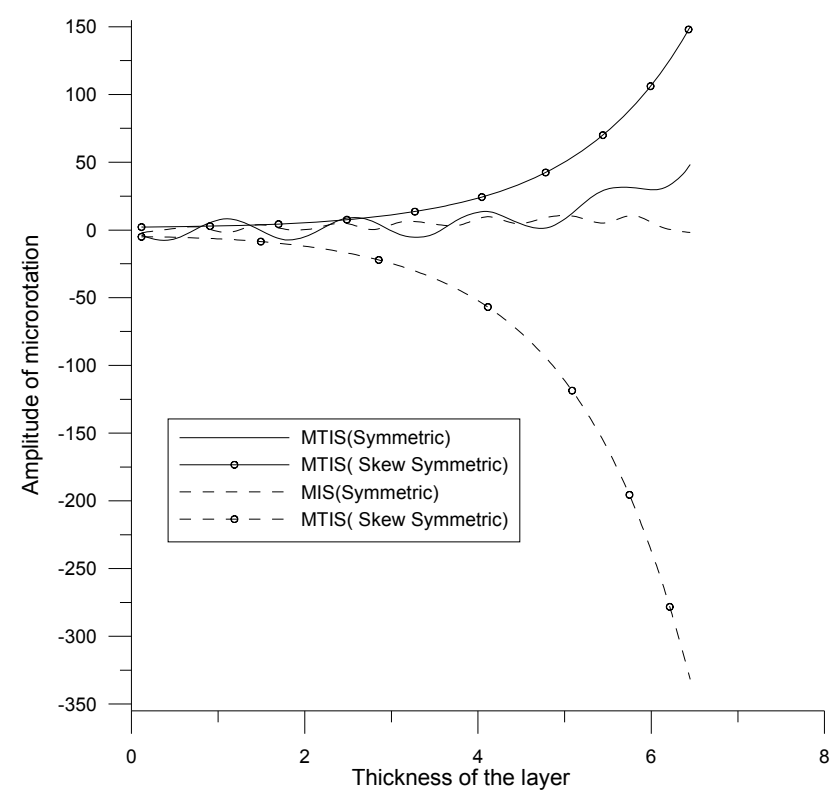

Fig.9. Variation of amplitude of microrotation with thickness $H$ of the layer. 


\section{Conclusions}

The propagation of circular crested waves in an infinite layer of a transversely isotropic medium after deriving the secular equation is investigated. The phase velocity for both symmetric and skew-symmetric modes attain larger values at vanishing wave number, which sharply flattens out to become steady with increasing wave number. An appreciable effect of anisotropy is evinced from all the curves. Also, its value becomes larger for the highest mode of wave propagation. The value of attenuation coeffcient and specific loss shows a similar behavior for both isotropic and anisotropic cases. The values of amplitude of radial displacement and microrotation get increased due to anisotropy for both symmetric and skew-symmetric cases, whereas, the amplitude of tangential displacement increases for the symmetric mode and gets decreased for the skew-symmetric mode.

\section{Nomenclature}

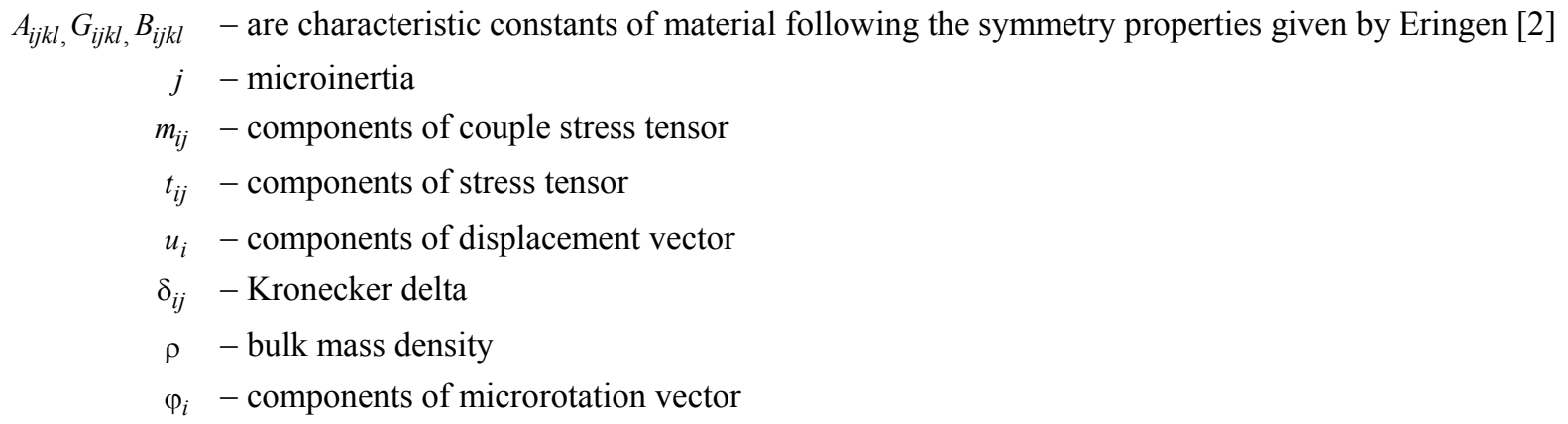

\section{References}

[1] Suhubi E.S. and Eringen A.C. (1964): Non-linear theory of simple microelastic solids II. International Journal of Engineering Sciences, vol.2, pp.389-404.

[2] Eringen A.C. (1999): Microcontinuum field theories I: Foundations and Solids. - New York: Springer Verlag.

[3] Mindlin R.D. (1964): Microstructure in linear elasticity. - Arch. Rational Mech. Anal., vol.16, pp.51-78.

[4] Eringen A.C. (1966): Linear theory of micropolar elasticity. - J. Math. Mech., vol.16, pp.909-923.

[5] Abubakar I. (1962): Free vibrations of a transversely isotropic plate. - Quarterly Journal of Mech. and Applied Math., XV, Pt.I, pp.129-136.

[6] Keck E. and Armenkas A.E. (1971): Wave propagation in transversely isotropic layered cylinders. - Journal of the Engg. Mech. Division Proceedings of the American Society of Civil Engg., EM 2, pp.541-555.

[7] Suvalov A.L., Poncelet O., Deschamps M. and Baron C. (1974): Long-wavelength dispersion of acoustic waves in transversely inhomogeneous anisotropic plates. - Wave Motion, vol.42, pp.367-382.

[8] Payton R.G. (1991): Wave propagation in a restricted transversely isotrpic elastic solid whose slowness surface contains conical points. - [Q.J.] Mech. Appl. Math., vol.45, Pt.2, pp.183-197.

[9] Eringen A.C. (1999): Microcontinuum field theories I: Foundations and Solids. - New York: Springer Verlag.

[10] Slaughter W.S. (2002): The linearized theory of elasticity. - Birkhauser.

[11] Parfitt and Eringen (1969): Reflection of plane waves from the flat boundary of a micropolar elastic half-space. The Journal of Acoustical Society of America, vol.45, No.5, pp.1258-1272.

[12] Kolsky H. (1963): Stress Waves in Solids. - New York: Clarendon Press, Oxford; Dover Press.

[13] Eringen A.C. (1964): Plane waves in nonlocal micropolar elasticity. - Int. J. Engng. Sci, vol.22, pp.1113-1121.

Received: October 17, 2017

Revised: August 13, 2018 